Relations industrielles

Industrial Relations

\title{
The Politics of Comprehensive Manpower Legislation, by Roger H. Davidson, Policy Studies in Employment and Welfare, Number 15, Baltimore, The John Hopkins Press, 1972, 118 pp.
}

\section{Jean Sexton}

Volume 28, numéro 3, 1973

URI : https://id.erudit.org/iderudit/028439ar

DOI : https://doi.org/10.7202/028439ar

Aller au sommaire du numéro

Éditeur(s)

Département des relations industrielles de l'Université Laval

ISSN

0034-379X (imprimé)

1703-8138 (numérique)

Découvrir la revue

Citer ce compte rendu

Sexton, J. (1973). Compte rendu de [The Politics of Comprehensive Manpower Legislation, by Roger H. Davidson, Policy Studies in Employment and Welfare, Number 15, Baltimore, The John Hopkins Press, 1972, 118 pp.] Relations industrielles / Industrial Relations, 28(3), 676-677.

https://doi.org/10.7202/028439ar

Tous droits réservés @ Département des relations industrielles de l'Université Laval, 1973
Ce document est protégé par la loi sur le droit d'auteur. L'utilisation des services d'Érudit (y compris la reproduction) est assujettie à sa politique d'utilisation que vous pouvez consulter en ligne.

https://apropos.erudit.org/fr/usagers/politique-dutilisation/ 
sultats. Par exemple, lorsque dix (10) municipalités contiguës possèdent le même grisé, est-ce à dire qu'elles ont toutes, chacune prise individuellement, le même nombre d'entreprises d'une certaine taille, ou plutôt que ce nombre s'applique à l'ensemble des municipalités ?

Sans doute cet atlas peut-il être utile à certains spécialistes dans des études spécifiques; je pense en particulier à des agents de développement industriel, à la recherche de sites possibles pour l'implantation de certaines entreprises. Il peut aussi fournir une bonne idée du déploiement de la main-d'oeuvre sur le territoire par secteur industriel. Je suis cependant moins confiant que l'auteur de cet instrument de travail, quant à l'utilité de celui-ci dans l'étude de la mobilité de la main-d'oeuvre. A cette fin, l'atlas devrait fournir une comparaison à deux moments du temps.

Personnellement, je souhaite, comme M. Marois lui-même, que cet atlas soit " un instrument de travail pour les chercheurs de toute discipline, qui s'intéressent à la main-d'oeuvre » (p. XIX). Je ne crois pas partager le même enthousiasme que M. Marois à cet égard.

\section{Bertrand BELZILE}

Université Laval

\section{The Politics of Comprehensive Man-} power Legislation, by Roger $\mathrm{H}$. Davidson, Policy Studies in Employment and Welfare, Number 15, Baltimore, The John Hopkins Press, 1972, 118 pp.

Depuis quelques années, les américains se préoccupent de plus en plus à décentraliser la politique de main-d'oeuvre et à en «décatégoriser》 les différents programmes. Alors que la très grande majorité des auteurs ne se contente que d'énoncer la nécessité d'une telle décatégorisation et décentralisation, très peu s'attardent à en présenter les difficultés pratiques.

Fondamentalement, cet ouvrage traite d'un des aspects de ce problème, peutêtre le plus important, à savoir l'aspect proprement politique des préoccupations en main-d'oeuvre. En effet, la mise en pratique d'une telle décatégorisation et décentralisation constitue en soi un changement tant dans la philosophie d'une politique de main-d'oeuvre que dans les structures des services aux clients. De tels changements doivent forcément être contenus dans des législations vu que les programmes à modifier sont déjà inclus dans des lois. Or c'est ici que surgit le problème politique et les conflits possibles entre technocrates spécialistes en ce domaine et les politiciens élus. Ce n'est que très rarement que ces gens sont en parfait accord sur ce qu'il y a à faire, pour des raisons évidentes. C'est pourquoi il arrive souvent qu'un projet de programme ou de loi préparé par des «spécialistes» ne soit plus reconnaissable comme tel après son adoption. Ce problème s'est présenté récemment aux Etats-Unis dans le domaine de la main-d'oeuvre. C'est ce que Davidson présente dans son livre.

Cet ouvrage caractérise en fait un nouveau courant de préoccupations en main-d'oeuvre, surtout en Amérique du Nord. En effet, la dernière décennie a été surtout marquée par l'étude des services à rendre suite à des travaux sur les imperfections du marché du travail. L'important a d'abord été de monter des programmes. Ce n'est en fait que vers la fin des années ' 60 que des préoccupations d'évaluation firent déborder les observateurs sur la nécessité de s'attarder plus longuement sur la façon dont ces services étaient rendus aux clients visés et sur leur coordination.

Fondamentalement, ce problème de coordination reste encore à résoudre et le demeurera encore un bon moment. La raison d'un tel problème semble résider dans le manque d'une acceptation universelle de la nature d'une politique de main-d'oeuvre.

L'ouvrage de Davidson a plusieurs mérites et quelques faiblesses. Débutons d'abord par les faiblesses.

L'auteur ne présente pas la conception de la politique de main-d'oeuvre selon laquelle il observe la réalité américaine et selon laquelle il fait ses suggestions. Ensuite, l'auteur s'attarde beaucoup trop longuement à faire la petite histoire du Comprehensive Manpower Act. Cela rend en fait la lecture du texte très monotone. Finalement, et cette remarque peut rejoindre notre premier point, les sugges- 
tions présentées ne découlent pas nécessairement et clairement de l'analyse.

Ce livre a cependant beaucoup plus de mérites et c'est ce qui en rend la lecture nécessaire à tous ceux qui s'intéressent au problème de la politique de main-d'oeuvre. D'abord, l'auteur met en lumière le problème vital des préoccupations de main-d'oeuvre dans une société industriclle avancée. Sur ce point, son message ressemble quelque peu à ce que Mangum et Levitan ont déjà avancé. En second lieu, Davidson dépeint très bien le fouillis administratif et organisationnel propre aux programmes américains de main-d'oeuvre. Il pose le problème d'une façon très intéressante. La citation suivante en est un exemple : « Program fragmentation is a product not only of agency rivalries but also, and more fundamentally, of the manner in which policies are formulated and sustained in a pluralist political system. As each new pressing need identified and publicized, a remedy or palliative is fashionned in the form of a governmental program. Thus government involvement tends to be a mosaic of singlepurpose efforts, with inevitable discard » (p. 7).

Ce premier chapitre dans lequel il présente le problème sous-étude est de loin le plus intéressant et le mieux fait du livre.

En troisième lieu, les préoccupations plus politiques de Davidson constituent le caractère original de ce travail. Cet aspect politique est en train de devenir, et avec raison, le sujet à la mode à l'intérieur des préoccupations de maind'oeuvre.

La plus grande leçon à tirer de ce livre par le lecteur non-américain réside sûrement dans l'apprentissage de ce qu'il ne faut pas faire pour organiser pratiquement des programmes de main-d'oeuvre. Les conflits entre niveaux de gouvernements, entre différents ministères et agences d'un même gouvernement et le fait que les préoccupations de main-d'oeuvre intéressent presque tout le monde exigent que la politique de main-d'oeuvre soit placée très haut dans la structure d'autorité. Espérer de la coordination entre plusieurs niveaux similaires d'autorité dans différents organismes peut être facilement une utopie. Cette coor- dination doit être forcée. C'est selon ce principe, sans que l'auteur le mentionne, qu'il en arrive à proposer une décentralisation sélective des programmes de main-d'oeuvre, décentralisation qui voit la définition des besoins et la livraison des services au niveau local, et la coordination, le financement, etc. à un niveau fédéral.

En résumé, ce livre présente des idées relativement récentes sur un sujet jusqu'ici très négligé mais crucial, à savoir la façon dont les services en main-d'oeuvre sont rendus. On sera sûrement appelé à réfléchir sur ce point bientôt au Canada et au Québec même si a priori plusieurs croient que de tels problèmes de coordination, de décentralisation et de «décatégorisation 》 ne se posent pas chez nous. Il sera alors sûrement utile de lire cet ouvrage de Davidson. En main-d'oeuvre, comme dans d'autres domaines, on a beaucoup à apprendre de l'expérience des autres.

\section{Jean SEXTON}

\section{Université Laval}

The Engineer in the Industrial Corporation, by Richard $R$. Ritti, New York, Columbia University Press, 1971, $261 \mathrm{pp}$.

On croit généralement que les laboratoires de recherche d'exploration et d'application constituent un repère où des individus hautement qualifiés peuvent encore exercer un certain contrôle dans l'organisation de leur travail et bénéficier ainsi de plus de latitude dans l'exercice de leurs fonctions. Cette croyance ne résiste pas complètement à l'observation des faits recueillis et analysés par Ritti dans douze laboratoires d'une grande entreprise américaine employant plus de 4,500 ingéneurs. Ritti, dans cet ouvrage, tente de dresser un profil de l'ingénieur malheureux sous deux aspects : celui de la sous-utilisation et du manque d'influence dans l'accomplissement de son rôle. Il recueille d'abord des données à l'échelle de l'entreprise et constate que la proportion des ingénieurs insatisfaits se rapproche de celle des techniciens et des ouvriers de la production. Il faut d'abord distinguer ces ingénieurs de ceux qui sont itinérants (field engineers) et des scientifiques qui présentent des profils spycho- 